\title{
332.
}

\section{ANALYTICAL THEOREM RELATING TO THE SECTIONS OF A QUADRIC SURFACE.}

[From the Philosophical Magazine, vol. xxvir. (1864), pp. 43, 44.]

THE four sections $x=0, y=0, z=0, w=0$ of the quadric surface

$$
a x^{2}+b y^{2}+6 x y \sqrt{a b}-c z^{2}-d w^{2}=0
$$

are each of them touched by each of the four sections

$$
x \sqrt{2 a}+y \sqrt{2 b} \pm z \sqrt{c} \pm w \sqrt{d}=0 ;
$$

where it is to be noticed that the radicals $\sqrt{2 a}, \sqrt{2 b}$ are such that their product is $=2 \sqrt{a b}$ if $\sqrt{a b}$ be the radical contained in the equation of the surface. There is of course no loss of generality in attributing a definite sign to the radical $\sqrt{2 a}$; but upon this being done, the sign of the radical $\sqrt{2 b}$ is determined, whereas the signs of $\sqrt{c}$ and $\sqrt{d}$ are severally arbitrary. We may if we please write the equation of any one of the last-mentioned sections in the form

$$
x \sqrt{2 a}+y \sqrt{2 b}+z \sqrt{c}+w \sqrt{d}=0,
$$

it being understood that the radicals $\sqrt{2 a}, \sqrt{2 b}$ have each a determinate sign, but that the signs of $\sqrt{c}$ and $\sqrt{d}$ are each of them arbitrary.

To prove the theorem in question, it is enough to show (1) that the sections $x=0$, $x \sqrt{2 a}+y \sqrt{2 b}+z \sqrt{c}+w \sqrt{d}=0 ; \quad$ (2) that the sections $z=0, \quad x \sqrt{2 a}+y \sqrt{2 b}+w \sqrt{d}=0$, touch each other. 
1. The sections $x=0, \quad x \sqrt{2 a}+y \sqrt{2 b}+z \sqrt{c}+w \sqrt{d}=0$ of the quadric surface $a x^{2}+b y^{2}+6 x y \sqrt{a b}-c z^{2}-d w^{2}=0$ will touch each other if, combining together the equations

$$
x=0, \quad y \sqrt{2 b}+z \sqrt{c}+w \sqrt{d}=0, \quad b y^{2}-c z^{2}-d w^{2}=0,
$$

these give a twofold value (pair of equal values) for the ratios $y: z: w$. We in fact have

$$
\begin{aligned}
b y^{2}-c z^{2}-d w^{2} & =b y^{2}-c z^{2}-(y \sqrt{2 b}+z \sqrt{c})^{2}, \\
& =-b y^{2}-2 c z^{2}-2 y z \sqrt{2 b c} \\
& =-(y \sqrt{b}+z \sqrt{2 c})^{2}
\end{aligned}
$$

and the right-hand side being a perfect square, the condition of contact is satisfied.

2. In like manner we have the system

$$
z=0, \quad x \sqrt{2 a}+y \sqrt{2 \bar{b}}+w \sqrt{d}=0, \quad a x^{2}+b y^{2}+6 x y \sqrt{a \bar{b}}-d w^{2}=0,
$$

which gives

$$
\begin{aligned}
a x^{2}+ & b y^{2}+6 x y \sqrt{a b}-d w^{2} \\
= & a x^{2}+b y^{2}+6 x y \sqrt{a b}-(x \sqrt{2 a}+y \sqrt{2 \bar{b}})^{2} \\
= & -a x^{2}-b y^{2}+2 x y \sqrt{a \bar{b}} \\
= & -(x \sqrt{a}-y \sqrt{b})^{2}
\end{aligned}
$$

and here also, the right-hand side being a perfect square, the condition of contact is satisfied.

Cambridge, November 28, 1863. 